\title{
Comunidades Virtuais em Redes Sociais na Internet: Uma proposta de estudo
}

\author{
Raquel da Cunha Recuero' \\ ECOS/UCPel e PPGCOM/UFRGS \\ raquel@pontomidia.com.br
}

\begin{abstract}
Resumo: $O$ trabalho visa expôr uma proposta de modelo para o estudo das comunidades virtuais em redes sociais na Internet. Tratase de um trabalho de cunho metodológico. Parte-se de uma definição sociológica do conceito de comunidade virtual e da construção interisciplinar do conceito de rede social. Por fim, com base neste debate, apresenta-se a proposta.
\end{abstract}

Palavras-Chaves: redes sociais, ciberespaço, comunidades virtuais.

Abstract: This paper deals with a model for the study of virtual communities as Internet social networks. It proposes a method of study for these phenomena. It starts from a conceptual definition of virtual communities and the interdisciplinary construction of the concepct of social network. Finally, based on this debate, the proposal is presented.

Keywords: social networks, cyberspace, virtual communities.

Résumén: Cet article traite de un modèle pour l'étude des communautés virtuelles comme réseaux sociaux dans l'Internet. Il propose une méthode d'étude pour cettes phénomènes Il commence à partir d'une définition conceptuelle des communautés et la construction interdisciplinaire du concepct du réseau social. En conclusion, basé sur cette discussion, la proposition est présentée.

Móts-Klés: réseaux sociales, cyberspace, communautés virtuelles.

\footnotetext{
${ }^{1}$ Doutoranda pelo Programa de Pós-Graduação em Comunicação e Informação da Universidade Federal do Rio Grande do Sul (PPGCOM/UFRGS) e professora da Escola de Comunicação da Universidade Católica de Pelotas (ECOS/UCPel). Pesquisadora vinculada ao Núcleo de Pesquisa em Comunicação (NUPECOM) da ECOS/UCPel e ao Grupo de Estudos de Interação Mediada por Computador (PPGCOM/UFRGS). O presente trabalho foi com o apoio do UOL - www.uol.com.br, processo número 200503101133.
} 
Extracto: Este papel se ocupa de un modelo para el estudio de comunidades virtuales como redes sociales del Internet. Propone un método de estudio para estos fenómenos. Empieza con una definición conceptual de las comunidades virtuales y de la construcción interdisciplinaria del concepct de la red social. Finalmente, basado en este discusión, se presenta la metodología.

Palabras claves: redes sociales, ciberespacio, comunidades virtuales.

\section{Introdução}

O presente trabalho propõe um modelo de estudo para as comunidades virtuais na Internet, a partir de estudos teóricos e práticos realizados em trabalhos anteriores (Recuero, 2002, 2003 a e 2003b, 2004 e 2005), em comunidades de weblogs e do Orkut. Busca-se uma sistematização dos elementos que devem ser observados no estudo das comunidades, tipos específicos de redes. O presente estudo faz parte de nossa tese de doutoramento e prevê ainda, desdobramentos futuros no sentido de validação e crítica do modelo.

\section{A Abordagem de Redes}

Embora questões relativas às redes tenham sido estudadas desde muito tempo ${ }^{2}$, recentemente, sua metáfora como forma de estudo das relações tem sido resgatada por diversos estudos em variados campos da ciência (Castells, 2003; Barabási, 2003; Watts, 2003 e 1999; Buchanan, 2002; Johnson, 2003) e denominada, muitas vezes como "a nova 3 ciência das redes" (Barabási, 2003; Thacker, 2004a e 2004b).

\footnotetext{
${ }^{2}$ Em 1736, Leonhard Euler resolveu o já famoso "Enigma das Pontes de Königsberg", na Prussia, criando a Teoria dos Grafos, um dos braços da Topologia. Um grafo é a representação topológica de uma rede, composta por vértices(ou nós) e arcos (ou arestas).

${ }^{3}$ Grifo meu.
} 
Esta abordagem apresenta uma busca por padrões comuns (denominados "padrões de rede”) a vários fenômenos de campos tão díspares quanto matemático, físico, econômico e social. Através da proposição de modelos de análise, seus teóricos clamam pela aplicabilidade destes em todos os campos da ciência, como as formas de compreender os "padrões de rede", de um modo especial, à própria Internet e às redes sociais que ali se constituem. Esses modelos seriam:

a) Modelo de Redes Aleatórias - Paul Erdös e Alfred Rényi, pensando sobre como se formariam as redes sociais, criaram o chamado "modelo de redes aleatórias" (Watts, 2003, 1999; Barabási, 2003; Buchanan, 2002). Eles acreditavam que o processo de formação das redes era randômico, no sentido de que esses nós se agregavam aleatoriamente. Dessa premissa, Erdös e Rényi concluíram que todos os nós, em uma determinada rede, deveriam ter mais ou menos a mesma quantidade de conexões, ou igualdade nas chances de receber novos links, constituindo-se, assim, como redes igualitárias.

b) Modelo de Mundos Pequenos - A partir do experimento de Milgram4 e das teorias de Granovetter5, Ducan Watts e Steven Strogatz (Watts, 1999 e 2003), descobriram que as redes sociais apresentavam padrões altamente conectados, tendendo a formar pequenas quantidades de conexões entre cada

\footnotetext{
${ }^{4}$ O sociólogo Stanley Milgram, na década de 60, foi o primeiro a realizar um experimento para observar os graus de separação entre as pessoas. (Dégene e Forsé, 1999; Buchanan, 2002; Barabasi, 2003 e Watts, 2003). Ele enviou uma determinada quantidade de cartas a vários indivíduos, de forma aleatória, solicitando que tentassem enviar a um alvo específico. Caso não conhecessem o alvo, as pessoas eram solicitadas então, a enviar as cartas para alguém que acreditassem estar mais perto dessa pessoa. Milgram descobriu que, das cartas que chegaram a seu destinatário final, a maioria havia passado apenas por um pequeno número de pessoas. Isso indicaria que as pessoas estariam efetivamente, a poucos graus de separação umas das outras Isso indiciaria que este seria, efetivamente, vivendo em um "mundo pequeno".

${ }^{5}$ Outra importante contribuição para o o problema da estruturação das redes sociais foi dada pelo sociólogo Mark Granovetter (1973). Em seus estudos, ele descobriu que chamou de laços fracos seriam muito mais importantes, na manutenção da rede social do que os laços fortes, para os quais habitualmente os sociólogos davam mais importância.
} 
indivíduo. Eles criaram um modelo semelhante ao de Erdös e Rényi, onde os laços eram estabelecidos entre as pessoas mais próximas e alguns laços estabelecidos de modo aleatório entre alguns nós transformavam a rede num mundo pequeno (Watts, 2003, 1999). Esse modelo demonstraria que a distância média entre quaisquer duas pessoas no planeta não ultrapassaria um número pequeno de outras pessoas, bastando que alguns laços aleatórios entre grupos existissem ${ }^{6}$ (Buchanan, 2002).

c) Modelo de Redes sem Escalas - O primeiro problema da teoria dos mundos pequenos de Watts foi explicado por Barabási (2003, p. 55-64) pouco tempo após a publicação do trabalho. Watts tratava as suas redes sociais como redes aleatórias, ou seja, redes em que as conexões entre os nós (indivíduos) eram estabelecidas de modo aleatório, exatamente como Erdös e Rényi anos antes. Entretanto, Barabási (2003) demonstrou que as redes não eram formadas de modo aleatório. Ele acreditava que, como os estudos de Watts e Strogatz, bem como de Granovetter tinham apontado, existia uma ordem na dinâmica de estruturação das redes, algumas leis bem específicas. Essa lei, ou padrão de estruturação, foi chamada por Barabási de "rich get richer"- ricos ficam mais ricos. Ou seja, quanto mais conexões um nó possui, maiores as chances de ele ter mais novas conexões. Ele chamou essa característica de conexão preferencial: Essa assertiva implica em outra premissa fundamental: As redes não seriam constituídas de nós igualitários, ou seja, com a possibilidade de ter, mais ou menos, o mesmo número de conexões. Ao contrário, tais redes possuriam nós que seriam altamente conectados (hubs ou conectores) e uma grande maioria de nós com poucas conexões. Os hubs seriam os "ricos", que tenderiam a receber sempre mais conexões. As redes

\footnotetext{
${ }^{6}$ Segundo Adamic e Adar (2004:2), é justamente a existência de atalhos entre os indivíduos em uma rede que caracteriza a existência desses mundos pequenos.
} 
com essas características foram denominadas por ele "sem escalas"7 (scale free).

Embora a "ciência das redes" seja relativamente recente, suas premissas podem ser encontradas em outros trabalhos bem mais antigos. $\mathrm{O}$ cerne dessas abordagens é, justamente, o foco no todo, mais do que nas partes e nas "interconexões" entre essas partes, em uma tentativa de observar os padrões que unem os elementos dos sistemas. Essas idéias são comuns à tradição da Teoria Geral dos Sistemas Bertalanffy (1975); e à Cibernética (Wiener, 2000; Ashby, 1970).

O estudo da sociedade a partir da perspectiva de rede, assim, remonta também ao estudos sistêmicos e aos modelos sociais baseados neles (Lakatos e Marconi, 1999, citam notadamente os modelos orgânico, de Spencer e Paretto, e processual como modelos "sistêmicos"). Um dos modelos mais famosos é o de Talcott Parsons (1969). O autor dizia que o sistema social deveria resolver quatro problemas básicos para a sua existência: adaptação, integração, conquista dos objetivos e manutenção dos padrões motivacionais e culturais.

Ao mesmo tempo, a Análise de Redes Sociais (ou Análise Estrutural de Redes Sociais) dedicou-se ao estudo da sociedade através da metáfora de rede vários anos antes da proposição da "ciência das redes". A Análise de Rede tem, também, um ramo sociológico, a Análise de Redes Sociais, baseada na Sociometria e na Teoria dos Grafos (Degenne e Forsé, 1999; Scott, 2001), que tem suas bases ainda nas décadas de 60 e 70 (Wellmann, 1989). Dentro desta perspectiva, uma rede social é compreendida como um conjunto de dois

\footnotetext{
${ }^{7}$ O nome "sem escalas" advém de características da representação matemática da rede. Matematicamente, as redes sem escala apresentam uma distribuição muito particular, denominada "power law" (uma curva logarítmica que descresce abruptamente a níveis próximos de um mínimo e mantém-se assim, construindo uma longa "cauda"). "A distribuição power law implica que uma abundância de nós possui apenas alguns links e uma minoria pequena, mas significativa, tem a grande maioria de links (Barabási, 2001, Tradução da autora: "...the power law distribution implies that there is an abundance of nodes with only few links, and a small-but significant-minority that have a very large number of links.")
} 
elementos: atores (pessoas, instituições ou grupos) e suas conexões (Wasserman e Faust, 1994, Degenne e Forsé, 1999). Essas conexões são entendidas como os laços e relações sociais que ligam as pessoas através da interação social. Trata-se de uma intersecção entre os modelos estruturaisfuncionalistas e os modelos matemáticos.

A aplicação direta dos referidos modelos da "ciência das redes" para os sistemas sociais não é capaz de dar conta da integralidade desses objetos. Embora em alguns estudos, os modelos propostos revelem-se frutíferos para análises dos fenômenos sociais ${ }^{8}$, questões essenciais permanecem. A perspectiva parece pressupor a conexão entre os atores de forma igual, sem salientar sua qualidade, sua profundidade e suas especificidades, que em redes sociais, podem fazer diferença. Sem levar em conta o custo do laço social e presumindo sua existência, os modelos podem levar a conclusões enganosas quando aplicados diretamente para os sistemas sociais. Outro senão é a incapacidade dos modelos de observar os vários sentidos nos quais as relações sociais acontecem, como o contexto e o capital social gerado fazem parte de cada interação em uma rede social. Os modelos também falham em levar em conta essas diferenças (Primo e Recuero, 2003 e 2004; Recuero, 2004). Notase que, enquanto a maioria dos problemas dos modelos teóricos da "ciência das redes" vem da falta de atenção para com a interação social, a abordagem sociológica supre esta lacuna com especial atenção para a estrutura. Entretanto, a abordagem sociológica, por demais formal, tem dificuldades para observar o estudo da dinâmica dessas redes, considerando-as como isoladas no tempo e espaço.

É neste intercâmbio que este trabalho posiciona-se. Trata-se de uma proposta de um modelo de estudo para as comunidades virtuais (compreendendo estas, como redes sociais) a partir de diálogos entre as teorias sistêmicas, cibernéticas, a ciência das redes e os estudos sociais.

\footnotetext{
${ }^{8}$ Vide, por exemplo, Newman, Watts e Strogatz (2002), Shirky (2003, online), Adamic e Adar (2004).
} 


\section{Redes Sociais na Internet: Organização, Estrutura e Dinâmica}

O estudo dos sistemas através de estrutura e organização é comum. Encontra-se em teóricos como Maturana e Varela (2001) e Talcott Parsons (1969), Galliano (1981). Neste trabalho, consideramos que a estrutura é aquilo que um grupo social tem de mais permanente, ou seja, implica em uma certa sedimentação dos modos de agir e das relações sociais. Já a organização trabalha com as relações de um modo geral, ou seja, o conjunto de elementos que faz parte da estrutura. Essas relações constituem-se na substância do extrato social. A estrutura, ao contrário, constitui-se naquilo que uma determinada sociedade possui para que seja considerada como tal.

Além desses dois elementos fundamentais, há a presença de um terceiro. As redes adaptam-se e modificam-se com o passar do tempo, como observou Bertalanffy (1975) em relação aos sistemas, explicando que tratamse de fenômenos processuais e históricos. A Cibernética também salientou essa característica demonstrando que os sistemas abertos perdem e adquirem energia do ambiente. Por isso, um modelo de rede que não leve em conta a dinâmica do sistema pode, invariavelmente, oferecer apenas uma "fotografia" de um fenômeno Thacker (2004a), estanque e parado no tempo. Deste modo, não se pode analisar uma rede unicamente sob as dimensões de estrutura e organização, pelo simples fato de que seu pressuposto fundamental é sua mudança no tempo. É necessário, portanto, incluir um novo item à análise: a dinâmica dos processos da rede social.

\section{1 . Organização: A Interação em Redes Sociais na Internet}

Ora, já foi dito que a organização constitui-se na totalidade de relações de um determinado agrupamento social. Neste sentido, pode-se dizer que a organização é composta pela interação social que constitui as relações de determinado grupo. Watzlavick, Beavin e Jackson (2000:46) explicam que a interação representa um processo sempre comunicacional. Neste sentido, a interação é aquela ação que tem um reflexo comunicativo entre o indivíduo e 
seus pares, como reflexo social (pg. 18). A interação, pois, tem sempre um caráter social perene e diretamente relacionado ao processo comunicativo.

No entanto, quando trabalhamos com o ciberespaço, a interação social dá-se de uma maneira muito particular. Trata-se de uma interação mediada pelo computador. Primo (1998 e 2003) estabelece uma dicotomia para tratar especificamente da interação mediada por computador. Para ele, existem unicamente duas formas de interação neste contexto: a interação mútua e a interação reativa. Estas formas distingue-se pelo "relacionamento mantido" (2003, p.61) entre os agentes envolvidos. Assim:

$$
\begin{aligned}
& \text { (...)interação mútua é aquela caracterizada por relações } \\
& \text { interdependentes e processos de negociação, em que cada } \\
& \text { interagente participa da construção inventiva e cooperada da } \\
& \text { relação, afetando-se mutuamente; já a interação reativa é } \\
& \text { limitada por relações determinísticas de estímulo e resposta } \\
& \text { (p.62). }
\end{aligned}
$$

A partir da dicotomia criada por Primo, pode-se imaginar que a interação social mediada por computador será sempre uma interação mútua. $\mathrm{Na}$ maioria das vezes, efetivamente, a interação reativa dá-se apenas entre o agente e o sistema que media a relação comunicativa (como no caso do link). Entretanto, em alguns casos, como no sistema do Orkut, é possível interagir com várias pessoas simplesmente através de botões, aceitando ou não uma amizade ou entrando para uma comunidade (Recuero, 2005). Embora essas interações não sejam mútuas, elas têm impacto social, já que têm também reflexos nos dois pólos da relação comunicativa. É claro que a interação reativa, por suas próprias limitações, acaba reduzindo o espectro de relações sociais que possa gerar e, conseqüentemente, de laços sociais. A interação mútua, por outro lado, como permite a inventividade, como explica Primo, pode gerar relações mais complexas do ponto de vista social.

Lakatos e Marconi (1999, p.88-93) salientam ainda outro aspecto importante: o da interação social como causadora de processos sociais diversos para um grupo, dependendo de sua natureza. Embora não seja 
explícito no trabalho das autoras, é perceptível que a interação social, a partir de suas naturezas diferenciadas, influencie os processos sociais que culminam em cooperação, competição e conflito. Esses processos serão tratados mais adiante, na parte de dinâmica do trabalho. Por hora, é importante salientar que a interação pode ter natureza diversa, ocasionando os referidos processos sociais.

É fundamental, portanto, para a análise da organização de uma rede social, a compreensão da interação mediada pelo computador em todos os seus aspectos. Elas proporcionam, portanto, também os processos dinâmicos da rede que serão discutidos adiante.

\subsection{Estrutura: Laços e Capital Social no Ciberespaço}

Como explicado, a estrutura da rede social compreende aquilo que ela possui de mais permanente, ou ainda, o resultado das interações repetidas. Trata-se de uma sedimentação dessas trocas, que pode ser observada através dos laços sociais e do capital social.

\subsubsection{Laço Social}

O conceito de laço social passa pela idéia de interação social, sendo denominado laço relacional, em contraposição ao laço associativo, aquele relacionado unicamente ao pertencer (a algum lugar, por exemplo)9 ${ }^{\text {. Os laços }}$ associativos constituem-se em meras conexões formais, que independem de ato de vontade do indivíduo, bem como de custo e investimento. Esses laços associativos podem emergir da existência dos laços sociais, constituindo-se num pertendimento relativo à existência de um grupo social mais denso, mas podem também representar apenas um mero reconhecimento formal da

\footnotetext{
${ }^{9}$ Breiger (1974: 183-185), inspirado nos trabalhos de Goffman (1971), explica que o laço social pode ser constituído de outra forma: através de associação. Goffman explicava que os individuos eram conectados a outros indivíduos através de relações sociais. Entretanto, a conexão entre um indivíduo e uma instituição ou grupo tornava-se um laço de outra ordem, representado unicamente por um sentimento de pertencimento. Tratava-se de um laço associativo.
} 
existência de um vínculo material entre um indivíduo e, digamos, um país. Neste sentido, não interferem na estrutura social, tratando-se, simplesmente, de uma classificação.

Os laços sociais também podem ser fortes e fracos Granovetter (1973). Laços fortes são aqueles que se caracterizam pela intimidade e pela proximidade. Os laços fracos, por outro lado, caracterizam-se por relações esparsas, que não traduzem proximidade e intimidade. Laços fortes constituem-se em vias mais amplas e concretas para as trocas sociais (Wellman, 1997), enquanto os fracos possuem trocas mais difusas. Evidentemente, os laços fortes constituem as redes menos instáveis, a estrutura mais forte de um determinado grupo, enquanto os fracos, os pontos de maior mutação.

Além disso, quanto maior o número de laços, maior a densidade da rede, pois mais conectados estão os indivíduos que fazem parte dela. Deste modo, os laços sociais auxiliam a identificar e compreender a estrutura de uma determinada rede social.

\subsubsection{Capital Social}

O capital social, de acordo com Putnam (2000, p.19), "refere-se à conexão entre indivíduos - redes sociais e normas de reciprocidade $e$ confiança que emergem dela"ı. Para o autor o capital social refere-se principalmente às conexões e tem como elementos a reciprocidade e a confiança.

Bourdieu também estabelece uma definição de capital social:

o capital social é o agregado dos recursos atuais e potenciais os quais estão conectados com a posse de uma rede durável, de relações de conhecimento e reconhecimento mais ou menos institucionalizadas, ou em outras palavras, à associação a um

\footnotetext{
${ }^{10}$ Tradução da autora para: "reffers to connection among individuals - social networks and the norms of reciprocity and trustwothiness that arise from them"
} 
grupo - o qual provê cada um dos membros com o suporte do capital coletivo (...) (1983, p.248-249) ${ }^{11}$.

Para o autor ${ }^{12}$ o capital social é relacionado, à um determinado grupo (rede social). O capital social, para Bourdieu, não se encontra nos indivíduos, mas ao contrário, encontra-se embutido nas relações sociais das pessoas.

Putnam (2000) e Bourdieu (1983) têm dois conceitos diferenciados. Para Putnam, o capital é um conjunto de recursos possuído pelo grupo, enquanto para Bourdieu, ele é uma conseqüência das relações sociais, que é percebida pelos envolvidos in abstracto (e que é, deste modo, passível de ser transformado por eles em outra forma de capital), mas pode ser construído e acessado apenas individualmente. Embora o capital social possa ser compreendido como um conjunto de recursos coletivos, que é "possuído" pelo grupo, é apenas através dos indivíduos que ele pode ser, efetivamente, construído e acessado.

Embora o capital social seja um conjunto de recursos coletivo, como salientado por Putnam, trata-se de recursos estes que estão embutidos nas relações sociais e, ao mesmo tempo, são definidos e moldados pelo conteúdo destas relações. O capital social existe, portanto, como um conjunto de recursos como o definido por Bourdieu. Ele apenas pode ser constituído e acessado de modo individual pelos atores, através do conteúdo de suas interações. Encontra-se, assim, embutido nessas mesmas interações, mas não restrito ao seu conteúdo, uma vez que este conteúdo pode variar, mas ainda assim, estar constituindo a mesma forma de capital social. Por conta disto, o

\footnotetext{
${ }^{11}$ Tradução da autora para: "social capital is the aggregate of the actual and potential resources which are linked to possession of a durable network of more or less institucionalized relationships of mutual acquaintance and recognition - in other words, to membership of a group - which provides each of the members with the backing of the collectivity-owned capital (...)".

${ }^{12}$ Para Bourdieu, o capital social é também referente ao campo de atuação (social). Outras formas de capital (econômico e cultural) seriam relacionadas a outros campos de atuação do indivíduo. Entretanto, o capital social pode ser convertido em outras formas de capital, como capital econômico, de acordo com as ações do grupo (1983, p. 249).
} 
capital social pode ainda ser acumulado, através do aprofundamento de um laço social (laços fortes permitem trocas mais amplas e íntimas), aumentando o sentimento de grupo.

Deste modo, o capital social constitui-se em um conjunto de recursos de um determinado grupo, obtido através da comunhão dos recursos individuais, que pode ser usufruido por todos os membros do grupo, ainda que individualmente, e que está baseados na reciprocidade. Ele está embutido nas relações sociais (como explica Bourdieu,1983) e é determinado pelo conteúdo delas (Gyarmati e Kyte, 2004; Bertolini e Bravo, 2004). Portanto, para que se estude o capital social dessas redes, é preciso estudar não apenas suas relações, mas igualmente, o conteúdo das mensagens que são trocadas através delas.

Bertolini e Bravo (2004:1-5) explicitam categorias que constituiriam aspectos nos quais o capital social pode ser encontrado. As categorias compreenderiam também os tipos de recursos que podem ser extraídos do capital social, a saber: a) relacional - que compreenderia a soma das relações, laçose trocasque conectam os indivíduos de uma determinada rede; b) normativo - que compreenderia as normas de comportamento de um determinado grupo e os valores deste grupo; c) cognitivo - que compreenderia a soma do conhecimento e das informações colocadas em comum por um determinado grupo; d) confiança no ambiente social - que compreenderia a confiança no comportamento de indivíduos em um determinado ambiente; e) institucional - que incluiria as instituições formais e informais, que constituem-se na estruturação geral dos grupos, onde é possível conhecer as "regras" da interação social, e onde o nível de cooperação e coordenação é bastante alto. Estes aspectos seriam ainda classificados como primeiro e segundo nível de capital social. O capital social de primeiro nível poderia ser associado com características individuais dos membros de uma rede, como as relações (a), leis ou normas (b) e conhecimento (c). Já o capital de segundo 
nível é aquele que só pode ser desfrutado por uma coletividade, portanto, sendo associado a um grupo e a uma maior sedimentação das relações, compreendendo a confiança no ambiente social (d) e a presença das instituições (e). A existência de capital social de primeiro nível é requisito para a constituição do capital de segundo nível (Bertolini e Bravo, 2004:5-10). Deste modo, um segundo nível de capital demonstra uma maior maturidade da rede social, além de maior densidade e existência no tempo de seus laços.

A estrutura de uma rede social na Internet pode ser estudada a partir dos dois aspectos: capital social e laços sociais. Entretanto, os laços sociais devem ser presumidos pela interação verificada. As interações podem demonstrar intimidade e seu acompanhamento pode mostrar que tipo de relação está sendo constituída. O estudo desses dois fatores permite, assim, que se compreenda como a rede social em questão está estruturada.

Por fim, na estrutura de uma rede social, podemos encontrar também a presença das estruturas previstas pelo modelos de rede da ciência das redes. É possível que as redes apresentem clusters (indivíduos altamente conectados), bem como mundos pequenos. Além disso, podem apresentar nós mais igualitários (com a mesma quantidade de conexões), o que também necessita ser avaliado.

\subsection{A Dinâmica da Rede Social}

Uma rede social, mesmo na Internet, modifica-se em relação ao tempo. Essas modificações constituem-se também em um padrão importante para a compreensão dessa rede (Thacker, 2004a e 2004b) e devem ser levadas em conta. Essas dinâmicas são dependentes das interações totais que abarcam uma rede (organização) e podem influenciar diretamente sua estrutura.

\subsubsection{Processos de Cooperação, Competição e Conflito}

Como já foi explicado anteriormente, Lakatos e Marconi (1999, p. 8893) trazem as interações como causadoras dos processos sociais de 
cooperação, conflito e competição.A cooperação seria o atuar em conjunto de um grupo ou de indivíduos, para a "consecção de um objetivo comum”, sendo, deste modo, essencial para a existência das estruturas sociais. Já a competição seria resultado das diferenças entre desejos e aspirações e a capacidade de provê-los ou ainda, a inadequabilidade desses recursos para toda uma população. A competição, embora não resulte necessariamente na constituição de novas estruturas sociais, não é também necessariamente, ponto de quebra dessas estruturas. Por fim, o conflito seria diferente da competição na medida em que envolve hostilidade e pessoalidade (Lakatos e Marconi, 1999, p. 89). Primo (2005), a partir de Simmel, salienta a importância de não se observar o conflito separadamente, de forma a percebê-lo unicamente como destruição da estrutura social. O autor salienta que "conflito e cooperação, por não serem extremos opostos, separados por um vazio abismal, só podem de fato ser separados conceitualmente” (2005, p. 20). De fato, as relações sociais podem ser constituídas de interações de natureza diversa. Entretanto, a diferenciação torna-se importante na medida em que auxilia a compreender os efeitos dessas interações sobre a estrutura de determinadas redes sociais. Não se pode, por exemplo, deduzir que não exista conflito em uma comunidade virtual. No entanto, é preciso que se compreenda que, para que a própria estrutura comunidade exista, a maioria das interações precisa ser cooperativa.

\subsubsection{Ruptura e Agregação}

Em estudos anteriores (Recuero, 2002) foi possível observar essa dinâmica em canais de chat. Quanto maior o número de pessoas que participavam do canal, maior a quantidade de conflitos e a formação de pequenos grupos. Os canais eram mais propensos a esses conflitos quando a interação era dificultada pela quantidade de pessoas conectadas. Quando um conflito entre grupos grandes acontecia, em geral, resultava em uma cisão, com a saída de um grupo para a formação de um "novo canal”. Do mesmo 
modo, grupos em weblogs e fotologs também são constantemente atacados e também passam por conflitos e rupturas. Allen (2004, online) trabalha com a possibilidade das rupturas acontecerem de modo "natural”. Ele explica que, de acordo com o trabalho de Dunbar, um antropólogo do University College of London, existiria um limite na quantidade de conexões que uma pessoa é capaz de manter ${ }^{13}$. Para Dunbar (1993), o limite seria biológico (referente ao tamanho do neocortex, região do cérebro), e seria o número de 150 (conhecido por “Dunbar number”), que Allen especifica como o tamanho máximo dos grupos com laços fortes. Dentro desta perspectiva, o processo de conflito seria tão importante quanto o de cooperação, para permitir que os grupos continuassem em tamanhos nos quais fosse possível a todos os seus membros interagir socialmente.

\subsubsection{Comportamentos Emergentes}

Outro aspecto importante da dinâmica das redes sociais é a emergência. Trata-se de uma característica dos sistemas complexos (Johnson, 2003) e envolve o aparecimento de padrões de comportamento em larga escala, que não são necessariamente determinados em micro-escala (Monge e Contractor, 2003). Dentro desta esfera, o aparecimento da ordem em sistemas caóticos, a auto-organização e a adaptação dos sistema, são considerados comportamentos emergentes. O próprio aparecimento de redes sociais na Internet pode ser considerado um comportamento emergente e autoorganizado. Características apontadas pelos modelos de Barabási (2003), como o aparecimento de clusters muito conectados em uma rede, e Watts (1999), como o aparecimento de "atalhos" nas redes sociais, constituindo pequenos mundos, também podem ser considerados padrões emergentes.

\footnotetext{
13 "There is a cognitive limit to the number of individuals with whom any one person can maintain stable relationships, that this limit is a direct function of relative neocortex size, and that this in turn limits group size ... the limit imposed by neocortical processing capacity is simply on the number of individuals with whom a stable inter-personal relationship can be maintained (Dunbar, 1993)"
} 
Redes sociais na Internet apresentam comportamentos emergentes com freqüência (Recuero, 2005), como a propagação de memes, a adaptação e a auto-organização, bem como o aparecimento de mundos pequenos e clusters (Shirky, 2003). É importante, entretanto, que se estude como a estrutura e a organização influenciam esses comportamentos.

\subsubsection{Adaptação e Auto-Organização}

Embora a adaptação dos sistemas sociais seja compreendida por muitos autores como Lakatos e Marconi (1999, p. 91-93) apenas do ponto de vista individual (adaptação do indivíduo à sociedade), interessa-nos neste tópico, a adaptação como processo coletivo. As redes adaptam-se ao ambiente. Maturana e Varela (2001) referenciam a adaptação como uma das características dos sistemas biológicos, definindo-as: "a manutenção dos organismos como sistemas dinâmicos em seu meio aparece como centrada em uma compatibilidade de organismo/meio. É o que chamamos de adaptação"(p. 115). Para os autores, essa é uma característica que implica na capacidade do sistema em auto-regular-se com relação às perdas de energia para o ambiente. A adaptação está relacionada ao acoplamento estrutural do organismo com o ambiente. O sistema está sempre adaptando-se ao ambiente, que produz alterações neste sistema que também, por sua vez, agem sobre esse ambiente. Essas alterações são causadas por perturbações recíprocas e recorrentes de um sistema sobre o meio e vice-versa. Neste fenômeno, existe uma contínua adaptação do ambiente ao sistema e do sistema ao ambiente, em uma relação circular.

O conceito de adaptação é diretamente relacionado ao de autoorganização, pensado pelos cibernéticos. Ashby (1970, p. 4), por exemplo, relaciona a idéia de auto-organização com o sistema nervoso. Ele explica que tratams-se de partes que organizam-se de forma a obter uma ordem que não pode ser encontrada em seu todo (sendo, portanto, também uma propriedade emergente). Von Foerster explica que sistema auto-organizador não apenas 
“importa ordem vinda de seu meio ambiente, mas também recolhe matéria rica em energia, integra-a em sua própria estrutura e, por meio disso, aumenta sua ordem interna"(Capra, 2000, p. 79).

Os sistemas sociais e as redes sociais, assim, estão em constante mudança. Essa mudança implica no aparecimento de novos padrões estruturais. Redes sociais, portanto, precisam ter capacidade de adaptação, pois têm um equilíbrio dinâmico, constantemente redirecionado entre caos e ordem.

\subsubsection{Sincronia}

A sincronia é apontado por Strogatz (2003) como uma forma de surgimento de "ordem espontânea" em um sistema complexo. Em outras palavras, a sincronia pode ser uma das formas de comportamento emergente em um determinado grupo social. Strogatz explica que ela acontece quando “as mesmas coisas acontecem ao mesmo tempo14" em vários sistemas ou grupos sociais(p.2) . Para o autor, a sincronia implica em uma ordem emergente, que não é acordada entre as partes do sistema, mas que simplesmente aparece nas interações coletivas, através do surgimento de um determinado ritmo. A sincronia seria ubíqua entre as coisas vivas, encontrada com persistência entre os chamados insetos sociais e outras formas de sistemas sociais no reino animal.

\subsubsection{Clusterização}

A clusterização é apontada, principalmente, pelo modelo de Barabási e Albert (1998). Barabási explica que há a presença de conectores em todas as redes, ou seja, indivíduos que possuiriam muito mais conexões com outras pessoas do que a média do grupo. A presença de conectores caracteriza o que se chama "clusterização" em uma determinada rede: O aparecimento de nós extremamente conectados. Essa presença de clusters em redes sociais afeta

\footnotetext{
${ }^{14}$ Tradução da autora para: "the same things happen at the same time".
} 
diretamente a teoria dos grafos randômicos de Erdös e Renyi. De acordo com essa teoria, os nós nas redes seriam formados de forma randômica e, portanto, possuiriam sempre um número mais ou menos semelhante de conexões, sendo chamadas "redes igualitárias". Barabási (2003, p. 62) demonstrou que a presença de clusters determinaria o que ele chama de "conexão preferencial" (através da metáfora rich get richer), ou seja, uma tendência a que os novos nós de uma rede conectem-se preferencialmente aos nós mais conectados (que ele chama de hubs). Além disso, Barabási explica que os hubs ou conectores teriam um papel fundamental nas redes sociais, porque seriam os responsáveis pela sua configuração como "mundo pequeno".

\section{Comunidades Virtuais como Redes Sociais}

Para discutir como a comunidade apareceria no modelo, é preciso, antes, discutir o que se entende pelo conceito. O termo "comunidade" é uma construção sociológica e evoluiu, através dela, de um sentido quase "ideal" de família, comunidade rural, passando a integrar um maior conjunto de grupos humanos com o passar do tempo (Tönies, 1995; Bottomore, 1989; Weber, 1987). Com o advento da modernidade e da urbanização, principalmente, as comunidades rurais passaram a desaparecer, cedendo espaço para as grandes cidades (Oldenburg, 1999; Rheingold, 1996). Com isso, a idéia de "comunidade" como sendo concebida como um tipo rural, ligado por laços de parentesco em oposição à idéia de sociedade, parece desaparecer, não da teoria, mas da prática. Rheingold (1996) diz que através do advento da Comunicação Mediada por Computador e sua influência na sociedade e na vida cotidiana, as pessoas estariam buscando novas formas de conectar-se, estabelecer relações e formar comunidades. Por isso, muitos autores optaram por definir as novas comunidades, surgidas no seio da CMC por "comunidades virtuais" (Rheingold, 1996; Donath, 1999; Wellman e Gulia, 1999).

Rheingold (1996, p. 20), um dos primeiros autores a efetivamente utilizar o termo "comunidade virtual", define-a: 
As comunidades virtuais são agregados sociais que surgem da Rede [Internet], quando uma quantidade suficiente de gente leva adiante essas discussões públicas durante um tempo suficiente, com suficientes sentimentos humanos, para formar redes de relações pessoais no espaço cibernético [ciberespaço] ${ }^{15}$.

De acordo com essa definição, os elementos formadores da comunidade virtual seriam: as discussões públicas; as pessoas que se encontram e reencontram, ou que ainda, mantêm contato através da Internet (para levar adiante a discussão); o tempo; e o sentimento. Esses elementos, combinados através do ciberespaço, poderiam ser formadores de redes de relações sociais, constituindo-se em comunidades. A comunidade virtual é, assim, um grupo de pessoas que estabelecem entre si relações sociais, que permaneçam um tempo suficiente para que elas possam constituir um corpo organizado, através da comunicação mediada por computador. No entanto, como compreender essas idéias com relação à rede social?

Um dos principais defensores de que as comunidades virtuais devam ser percebidas e estudadas como redes sociais é Barry Wellman. Em diversos trabalhos (Wellman e Gulia,1999; Wellman 2001, 2000), o autor destaca a importante transição ocasionada pelo desenvolvimento dos meios de comunicação e de transporte na sociabilidade humana ${ }^{16}$. Com isso, Wellman defende a idéia de que a transição entre a comunidade e a rede se deu no decorrer do tempo e não com o surgimento da Internet. Castells (2003) compartilha do entendimento de Wellman. Para o autor, "a comunidade desloca-se para a rede como a forma central de organizar a interação" (p.106). A diferença básica é que as redes seriam montadas pelas ações

\footnotetext{
15 Tradução da autora. No original: "Las comunidades virtuales son agregados sociales que surgem de la Red cuando una cantidad suficiente de gente lleva a cabo estas discusiones públicas durante un tiempo suficiente, com suficientes sentimentos humanos como para formar redes de relaciones personales en el espacio cibernético.”

${ }^{16} \mathrm{Na}$ esteira de McLuhan, trata-se de dizer que a vila de um indivíduo torna-se global.
} 
(escolhas, intenções) dos atores sociais. Isso implicaria nos laços sociais serem mais seletivos, formados de acordo com os interesses das pessoas.

Garton et. al (1997, p.5) defendem que é possível encontrar, em redes, padrões específicos de relações, que seriam associados aos grupos sociais. Esses padrões seriam referentes aos modos de relações entre os atores da rede. Eles explicam que esses padrões auxiliam o cientista a encontrar quem pertence e quem não pertence a um determinado grupo. Além disso, através da CMC é possível seguir a interação entre os atores de uma rede (uma vez que é possível acompanhá-la publicamente em vários sistemas, como em um canal de chat - onde se pode, inclusive, gravar as interações - ou em comentários de blogs - que ficam registrados, a menos que o autor deseje apagá-los), identificando grupos com conexões mais ou menos formais. Garton et. al. também salientam que é possível, através da CMC estudar a evolução de um determinado conjunto de atores (ou grupo) no tempo, justamente, devido ao registro da interação. Por fim, os autores sustentam também que é possível observar-se que tipo de recurso é trocado através dessa interação, para melhor identificar a estrutura da rede.

Então, como compreender a comunidade virtual na rede social?

Inicialmente, a comunidade é um grupo de pessoas que interage. No ciberespaço, esta interação se dá via comunicação mediada por computador e de forma mútua. A interação que acontece dentro de uma determinada rede é a base do estudo de sua organização. Ela pode ser cooperativa, competitiva ou geradora de conflito. A interação que é cooperativa pode gerar a sedimentação das relações sociais, proporcionando o surgimento de uma estrutura. Quanto mais interações cooperativas, mais forte se torna o laço social desta estrutura, podendo gerar um grupo coeso e organizado. Na organização da comunidade virtual, portanto, é necessário que exista uma predominância de interações cooperativas, no sentido de gerar e manter sua estrutura de comunidade. 
A estrutura da comunidade, no entanto, poderá ser analizada a partir dos laços e capital social verificados atrasvés da interação. Espera-se que a comunidade possua um núcleo com laço fortes, que consistirá no grupo mantenedor da estrutura. Essas pessoas têm muitos laços entre si, e laços fracos em torno de si, consistindo outros indivíduos que poderão ser agregados ao núcleo ou não. Além disso, espera-se que uma comunidade tenha grandes quantidades de capital social de primeiro e segundo nível, demonstrando também, a sedimentação dos recursos. Também a presença do interesse comum, como fundamento da pode indicar a possibilidade de tal grupo constituir-se em uma comunidade virtual, já que estabelece a cooperação como princípio.

Por fim, a dinâmica da comunidade deve ser adaptativa, autoorganizada e cooperativa. Além disso, padrões de sincronismo e clusterização são esperados, pois demonstram a coesão estrutural do grupo. Também a agregação de novos membros é esperada, pois trata-se de elemento fundamental para o crescimento e manutenção da rede. Essas dinâmicas, entretanto, podem não ser específicas das comunidades virtuais, mas comuns a várias redes sociais.

\section{Conclusão e Futuros Trabalhos}

O presente artigo buscou levantar elementos que devem ser considerados no estudo de uma comunidade virtual como rede social na Internet. Trata-se de uma sistematização teórico-prática, resultado das observações realizadas em trabalhos anteriores, e que é parte de nossa tese de doutoramento. Neste trabalho, defendemos a idéia de que as redes sociais no ciberespaço podem ser estudadas através de três grandes elementos: sua estrutura, sua organização e sua dinâmica. Dentro desses elementos, identificamos o que poderia ser compreendido como parte de uma rede social e quais elementos deveriam ser levados em conta no ciberespaço, a saber: na organização, as interações gerais do grupo; na estrutura, os laços e o capital 
social, principalmente, embora outras características previstas nos modelos de Barbási e Albert (2003), Watts e Strogatz (Watts, 1999 e 2003) e Ërdos e Rényi (Buchanan, 2002) possam ser encontrados; na dinâmica, os processos de cooperação, competição e conflito, agregação e ruptura e os comportamentos emergentes (auto-organização, adaptação, propagação de memes, etc.). Dentro desses elementos levantou-se, a partir da discussão teórica do conceito, quais poderiam ser encontrados nas comunidades virtuais. A partir desta abordagem teórico-metodológica, em trabalhos seguintes, pretende-se demonstrar os resultados da aplicação para as redes sociais na Internet e as comunidades virtuais.

\section{Referências:}

ADAMIC, Lada; ADAR, Eitan. How to Search a Social Network. Disponível em <http://www.hpl.hp.com/research/idl> Acesso em 23/03/2004.

ALLEN, Christopher. The Dunbar Number as a Limit to Group Sizes. Publicado em10/03/2004. Disponível em < http://www.lifewithalacrity.com/2004/o3/the_dunbar_numb.html> Acesso em 05/03/2005.

ASHBY, W. Ross. Introdução à Cibernética. São Paulo: Editora Perspectiva, 1970.

BARABÁSI, Albert-László et al. Evolution of the Social Network of Scientific Collaborations. Physica A, 311. 2002. pág. 590-614.

BARABÁSI, Albert. Linked. How Everything is Connected to Everything else and What it means for Business, Science and Everydai Life. Cambridge: Plume, 2003. 
BERTOLINI, Sandra e BRAVO, Giácomo. Social Capital, a

Multidimensional Concept. Disponível em

<http://www.ex.ac.uk/shipss/politics/research/socialcapital/other/bertol ini.pdf $>$ Acesso em 17/10/2004.

BERTALANFFY, Ludwig Von. Teoria Geral dos Sistemas. Petrópolis: Vozes, 1975. $2^{\mathrm{a}}$ edição.

BREIGER, Ronald. The Duality of Persons and Groups. Social Forces, Volume 53, Special Issue (Dec. 1974), pp.181-190.

BERTALANFFY, Ludwig Von. Teoria Geral dos Sistemas. Petrópolis: Vozes, 1975. $2^{\mathrm{a}}$ edição.

BOTTOMORE, T. B. Introdução à Sociologia. Rio de Janeiro: LTC - Livros Tecnicos e Científicos Editora S.A, 1987. 9 $9^{\mathrm{a}}$. edição.

BOURDIEU, Pierre. The Forms of Capital. Originalmente publicado em "Ökonomisches Kapital, kulturelles Kapital, soziales Kapital” in Soziale Ungleichheiten (Soziale Welt, Sonderheft 2). (pp. 248-257) Tradução de Richard Nice. Disponível online em <http://www.pontomidia.com.br/raquel/resources/o3.html>. Acesso em 15/07/2004.

BUCHANAN, Mark. Nexus: Small Worlds and the Groundbreaking Theory of Networks. New York: W.W. Norton e Company, 2002.

CASTELLS, Manuel. A Galáxia da Internet. Rio de Janeiro: Jorge Zahar editora, 2003.

DEGENNE, Alain e FORSÉ, Michel. Introducing Social Networks. London: Sage, 1999.

DONATH, Judith S. Identity and Deception in the Virtual Community. In KOLLOCK Peter. e Marc Smith. (organizadores) Communities in Cyberspace. Routledge. New York, 1999. pp. 29-59

DUNBAR, R. I. M. (1993). Coevolution of neocortical size, group size and language in humans. Behavioral and Brain Sciences 16 (4): 681735. Disponível em <http://www.bbsonline.org/documents/a/oo/oo/05/65/bbsoooo0565oo/bbs.dunbar.html >.Acesso em 05/03/2005.

GALLIANO, A. G. Introdução à Sociologia. São Paulo: Harper e Row do Brasil, 1981.

GARTON, Laura; HARTHORNTHWAITE, Caroline; WELLMAN, Barry. Studying Online Social Networks. Journal of Computer Mediated Communication, V 3, issue 1 (1997). Disponível em <http://www.ascusc.org/jcmc/vol3/issue1/garton.html>. Acesso em 12/04/2004. 
GYARMATI, David e KYTE, Darrel. Social Capital, Network Formation and the Community Employment Innovation Project. In: Policy Research Iniciative, Volume 6, Number 3. Disponível em $<$ http://policyresearch.gc.ca/page.asp?pagenm=v6n3_art_05>. Acesso em 04/05/2004.

GRANOVETTER, Mark. The Strength of Weak Ties. In The American Journal of Sociology, Vol. 78, No. 6, Maio de 1973. pp 1360-1380.

JOHNSON, Steve. Use the blog, Luke. Revista Salon, 10/05/2002. Disponível em <http://www.salon.com/tech/feature/2002/05/10/blogbrain/>. Acesso em 15/05/2002.

LAKATOS, Eva Maria e MARCONI, Marina de Andrade. Sociologia Geral. São Paulo: Atlas, 1999. $7^{\mathrm{a}}$. edição.

MATURANA, Humberto e VARELA, Francisco. A Árvore do Conhecimento. São Paulo: Palas Athena, 2001.

NEWMAN, M. E. J. Small Worlds. The Structure os Social Networks. Disponível em <http://citeseer.ist.psu.edu/514903.html > (Acesso em 2004)

NEWMAN, M. E. J., WATTS, Duncan e STROGATZ, Steve. Randon Graph Models of Social Networks. Publicado em Fevereiro de 2002. PNAS, vol. 99, pp. 2566-2572. Disponível em <http://www.pnas.org/>.Acesso em 04/12/2004.

OLDENBURG, Ray. The Great Good Place. New York: Molwe \&Company, 1999.

PARSONS, Talcott. Sociedades: Perspectivas Evolutivas e Comparativas. São Paulo: Livraria Pioneira Editora, 1969.

PRIMO, Alex. Interação Mútua e Interação Reativa: Uma proposta de Estudo. Trabalho apresentado no XXI Congresso da Intercom em setembro de 1998. Recife, PE. Disponível em <http://usr.psico.ufrgs.br/ aprimo/pb/intera.htm>. Acesso em 06/01/2005.

. Conflito e cooperação em interações mediadas por Computador.Trabalho apresentado no GT de Tecnologias Informacionais da Comunicação da Compós. Niterói, RJ, 2005.

Interação Mediada por Computador: a comunicação e a educação a distância segundo uma perspectiva sistêmicorelacional. Tese de Doutorado. Apresentada ao Programa de PósGraduação em Informática na Educação em março de 2003.

PRIMO, Alex e RECUERO, Raquel. Hipertexto Cooperativo: Uma análise da escrita coletiva a partir dos blogs e da wikipedia. Revista da FAMECOS, Porto Alegre, v. 22, p. 54-65, 2003. 
. Co-Links: Proposta de uma nova

tecnologia para a escrita coletiva de links multidirecionais.

Trabalho apresentado no GT de Tecnologias Informacionais da

Comunicação da COMPÓS. São Bernardo do Campo: 2004.

. Conflito e cooperação em

interações mediadas por Computador.Trabalho apresentado no GT de Tecnologias Informacionais da Comunicação da Compós. Niterói, RJ, 2005 .

PUTNAM, Robert D. Bowling Alone. The collapse and Revival of American Community. New York: Simon e Schuster, 2000.

RADICCHI, Filippo et. al. Defining and Identifying Communities in Networks. Publicado em 02/03/2004. PNAS, vol. 101, no. 9, pp. 26582663. Disponível em <http://www.pnas.org > . Acesso em 12/12/2004.

RECUERO, Raquel da Cunha. Teoria das Redes e Redes Sociais na Internet. Trabalho apresentado no XXVII INTERCOM, na PUC/RS em Porto Alegre. Setembro de 2004.

\section{. Weblogs, Webrings e}

Comunidades Virtuais. Revista 404notfound - Revista Eletrônica do Grupo Ciberpesquisa. Edição 31, agosto de 2003a. Disponível em <http://www.facom.ufba.br/ciberpesquisa/404nOtFound/404_31.htm> . Webrings: As Redes de Sociabilidade e os Weblogs. Revista Sessões do Imaginário, da Famecos/PUCRS. $2004 b$.

. Um estudo do capital social gerado a partir de redes sociais no Orkut e nos Weblogs. Trabalho apresentado no GT de Tecnologias Informacionais da Comunicação da Compós. Niterói, RJ, 2005.

Comunidades Virtuais no IRC: o caso do \#Pelotas. Um estudo sobre a Comunicação Mediada por Computador e a estruturação de comunidades virtuais. Dissertação apresentada como requisito parcial para a obtenção do título de mestre em Comunicação e Informação pela Universidade Federal do Rio Grande do Sul. (Janeiro de 2002). Disponível em

<http://www.pontomidia.com.br/raquel/dissertacao.pdf>

SCOTT, John. Social Network Analysis. A Handbook. London UK: Sage Publications, 2000. Second Edition.

SHIRKY, Clay. Power Laws. Weblogs and Inequality. Publicado em 10/02/2003. Disponível em <http://www.freerepublic.com/focus/fnews/840997/posts $>$. Acesso em 23/03/2004. 
STROGATZ, Steven. Sync. The emerging science of spontaneous order. New York: Theia, 2003.

TINDALL, D. B; WELLMAN, Barry. Canada as Social Structure: Social Network Analysis and Canadian Sociology. Journal of Canadian Sociology, 2001. Disponível em <http://www.chass.utoronto.ca/ wellman/publications/cansocstr/Can_ Net-Final-3oAp01.htm>. Acesso em 22/03/2004.

TÖNIES, Ferdinand. Comunidade e Sociedade. Textos Selecionados. In: MIRANDA, Orlando. Para Ler Ferdinand Tönies. São Paulo: Edusp,1995.

VON FOESTER, Heinz. On Constructing a Reality. Palestra proferida na Fourth International Conference on Environmental Design Research no dia 15 de Abril de 1973, na Virginia Polytechnic Institute em Blacksburg, Virginia. Foi publicado em: Heinz von Foerster, Observing Systems, Intersystems Publications 1984. 288-309. Disponível para acesso em <http://www.unikk.ch/course/Seiten/lesson2.2.htm>. Acesso em 08/09/2004.

WASSERMAN, Stanley e FAUST, Katherine. Social Network Analysis. Methods and Applications. Cambridge, UK: Cambridge University Press, 1994.

WATZLAVICK, Paul, BEAVIN, Janet e JACKSON, Don. Pragmática da Comunicação Humana. São Paulo: Cultrix, 2000. 11 ${ }^{\mathrm{a}}$. edição.

WATTS, Duncan J. Six Degrees. The Science of a Connected Age. New York: W. W. Norton \&Company, 2003.

. Small Worlds. The dynamics of Networks between Order and Randomness. New Jersey: Princetown University Press, 1999.

WEBER, Max. Conceitos Básicos de Sociologia. São Paulo: Editora Moraes, 1987.

WELLMAN, Barry. Physical Place and CyberPlace: The Rise of Personalized Networking. Fevereiro de 2001. Disponível em: $<$ http://www.chass.utoronto.ca/ wellman/publications/individualism/ij urr3a1.htm>. Acesso em abril de 2004.

. Structural Analysis: From Method and Metaphor to Theory and Substance. Publicado em: WELLMAN, Barry e BERKOWITZ, S. D. editores. Social Structures: A Network Approach. Cambridge: Cambridge University Press, 1988.

\section{. An Electronic Group is Virtually a Social}

Network. In KIESLER, Sarah (org.) Culture of Internet. Hilsdale, NJ: Lawrence Erlbaum, 1997. pp. 179-205. 
WELLMAN, Barry e GULIA, Milena. Net Surfers don't Ride Alone: Virtual Communities as Communities. Publicado em 1999. Disponível em <http://www.acm.org/ ccp/references/wellman/wellman.html>. Acesso em 05/05/2005.

WIENER, Norbert. Cibernética e Sociedade: O Uso Humano de Seres Humanos. São Paulo: Cultrix, 2000. $7^{\mathrm{a}}$. Edição.

WORTLEY, Scott e WELLMAN, Barry. Different Strokes from Different Folks: Community Ties and Social Support In: American Journal of Sociology 96, Nov., 1990 (p. 558-88).

THACKER, Eugene. Networks, Swarms and Multitudes $<$ http://www.ctheory.net/text_file.asp?pick $=422>$ (a) (parte 1) e $<$ http://www.ctheory.net/text_file.asp?pick=423> (b) (parte 2). Publicado em 18/5/2004. Acesso em 06/04/2005. 